\title{
Special issue on sustainable computing systems and applications
}

\author{
Danilo Ardagna ${ }^{1}$. Cinzia Cappiello
}

ICT and Green are two tightly related concepts. ICT systems in fact might support sustainability in different ways. On the one hand, ICT can be used to monitor the impact of other systems (e.g., buildings, transportation) on the environmental sustainability and to support green-aware decisions. On the other hand, the design of more efficient ICT systems can contribute in decreasing the energy consumption related to ICT itself, that is continuously increasing. In some regions of the world (e.g., USA and UK) the energy consumed by the data centers is close to $10 \%$ of the total energy consumed by the country. For example, Facebook data centers consumed overall 822 million of KWh in 2013. The company is particularly committed to reduce environmental impact, since our Facebook use for the entire year has about the same carbon impact as a making a medium cappuccino.

This Special Issue presents recent innovative works that deal with the design of Green ICT systems and covers topics ranging from green principles to the design and operation of software-intensive systems, the monitoring of data centers and the dynamic management of their infrastructure.

These open challenges raise a lot of interest throughout the scientific community. Fifteen high quality papers were submitted, out of which we selected three papers (approximately 20\% acceptance ratio) authored by Dr. Erik Jagroep, Jan Martijn E. M. van der Werf, Sjaak Brinkkemper, Leen Blom, Rob van Vliet, Claudia Canali, Riccardo Lancellotti, and Jordi Guitart.

Danilo Ardagna

danilo.ardagna@polimi.it

Cinzia Cappiello

cinzia.cappiello@polimi.it

1 Department of Electronic Information and Bioengineering, Politecnico di Milano, P.za Leonardo Da Vinci 32, 20133 Milan, Italy 
In particular the work by Jagroep et al. proposes a methodology to analyse the software architecture and identifies the actual drivers behind software components energy consumption. Canali and Lancellotti face the problem of VMs placement in virtualized infrastructures and propose a novel approach, based on monitoring and VMs clustering, to identify relevant VMs classes to solve efficiently and at scale the placement problem. Finally, Guitart's work proposes a holistic approach and a management architecture for sustainable data centers identifying design guidelines to reduce the carbon footprint of cloud systems.

We would like to thank the authors of all the submitted papers for the high quality of their scientific contributions. We also thank all reviewers for their dedication and timeless efforts, which allowed us to select very high quality papers. 